\title{
Double Regulation of Tonifying-Detoxifing on Elderly Diabetic Coronary Heart Disease After PCI
}

\author{
Fu Xianzhao*, Jiang Xiaofeng, Chen Jiajun, Cen Qianli, Li Yuegui, Qiu Haixian
}

The Affiliated Hospital of Youjiang Medical University for Nationalities, Baise, China

\author{
Email address: \\ 1620100638@qq.com (Fu Xianzhao) \\ ${ }^{*}$ Corresponding author
}

\section{To cite this article:}

Fu Xianzhao, Jiang Xiaofeng, Chen Jiajun, Cen Qianli, Li Yuegui, Qiu Haixian. Double Regulation of Tonifying-Detoxifing on Elderly Diabetic Coronary Heart Disease After PCI. American Journal of Biomedical and Life Sciences. Vol. 9, No. 5, 2021, pp. $286-291$. doi: $10.11648 /$ j.ajbls.20210905.20

Received: October 2, 2021; Accepted: October 26, 2021; Published: November 5, 2021

\begin{abstract}
Objective to investigate the effect of Huoxue Jiedu Jiangtang Recipe (HJJR) on cardiac remodeling and function prognosis in diabetic patients complicated with coronary heart disease (CHD) after percutaneous coronary intervention (PCI), and to explore its possible mechanism. Methods 92 diabetic patients with CHD who had received PCI therapy, were equally randomized into the treatment group (46) and the control group (46) from January 2016 to August 2016. The control group were given standardized western medicine, while treatment group received HJJR besides standardized treatment. The treating course were 2 months. Before and after treatment, the left ventricular morphology index [left ventricular end diastolic diameter (LVEDD), left ventricular end systolic diameter (LVESD), left ventricular posterior wall thickness (LVPWT), left ventricular end-diastolic ventricular septal thickness (LVEST)] and the cardiac function data [stroke volume (SV), left ventricular fractional shortening (FS), the cardiac index (CI), cardiac output (CO), maximum blood flow velocity of early diastolic/ atrium late diastolic (E/A), left ventricle ejection fraction (EF)] were detected with echocardiography. The serum levels of C-reactive protein (CRP), tumor necrosis factor-a (TNF- $\alpha$ ), interleukin-6 (IL-6) and Angiotensin II (Ang II) were tested with enzyme linked immunosorbent assay (ELISA), and the clinical efficacy and 5-year survival rate were evaluated. Results The total clinical effective rate and 5 -year survival ratio in treatment group were higher than those in control group $(\mathrm{P}<0.05)$. Serum inflammatory mediators (CRP, IL-6, TNF- $\alpha$ ) and Ang II decreased more than that in the control group (all P<0.05); And in treatment group, the left ventricular morphology index (LVESD, LVEDD) decreased more than that in the control group, while the cardiac function indexes ( $\triangle \mathrm{FS}, \mathrm{CO}, \mathrm{CI}, \mathrm{SV}, \mathrm{LVEF}, \mathrm{E} / \mathrm{A})$ were increased more in treatment group. Conclusion HJJR can hold back the deterioration of heart restructure, improve heart function, ameliorate prognosis and prolong living in diabetic CHD after PCI, and its partial therapeutic effects might come from its reducing the level of Ang II and anti-inflammatory function.
\end{abstract}

Keywords: Huoxue Jiedu Jiangtang Recipe, Diabetes Mellitus, Coronary Heart Disease, Heart Remodeling, Percutaneous Coronary Intervention

\section{Introduction}

Since 1977, when Swiss scientist Cruentzig succeeded in opening the narrow coronary artery by percutaneous coronary intervention (PCI) for the first time, creating a new era in the medical history for treating coronary heart disease (CHD), PCI has made rapid development, becoming an effective treatment, and widely applied to the reperfusion treatment of CHD. However, the severity of coronary artery disease is related to a variety of risk factors, among which diabetes is the most significant independent risk factor. CHD with diabetes usually presents multiple stenosis and often coexist with microvascular disease [1]. Generally, non-diabetic patients have a compensatory dilative movement in the non-infarcted vascular after the onset of myocardial infarction, while in diabetic patients, due to multiple vascular and myocardial microvascular lesions, the vascular compensatory movement is weakened [2]. Therefore, even if PCI is performed in time to restore myocardial perfusion and obtain temporary remission, the progress of diabetic cardiac remodeling is faster than that of non-diabetic patients. The important goal of heart failure treatment is to prevent and delay the development of 
myocardial remodeling, so as to reduce the mortality and hospitalization rate, not to improve temporary symptoms and quality of life only [3]. At present, with the deepening of clinical research, the maturity of technology and the continuous improvement of interventional materials, the indications of PCI treatment are expanding. However, for diabetic CHD, the problems faced by stenting will be increasingly complex, and the residual risk of Western medicine treatment will continuously accumulate. According to the theory of traditional Chinese medicine (TCM), diabetes belongs to the category of Xiao Ke, whose basic pathogenesis is the deficiency of both Qi and Yin, coexist with the accumulation of dry-heat. The deficiency of Qi can cause blood stasis, while the Yin deficiency can intensify dry-heat, and then the accumulation of heat-stasis can finally change into toxin. Therefore, "deficiency, blood stasis and toxin" are the important pathogenic factors of diabetic CHD [4]. Previous studies have shown that, the combination of Huoxue Jiedu Jiangtang Recipe (HJJR), which has the function of Nourishing Yin, benefiting Qi, activating blood and detoxifying, and Western Medicine, can significantly regulate the anti-inflammatory/ pro-inflammatory balance, effectively inhibit the inflammatory response, improve the myocardial blood supply [5]. The purpose of this study is to further explore the effect of HJJR on the cardiac remodeling after PCI in patients with diabetic acute coronary syndrome (ACS), and to search the effective method of the therapeutic complementary of Chinese and Western medicine, to delay and reduce the death rate of diabetes mellitus.

\section{Materials and Methods}

\subsection{Diagnostic Criteria}

\subsubsection{Diagnostic Criteria of Western Medicine}

Diabetes mellitus meets the diagnostic criteria of Chinese guidelines for the prevention and treatment of type 2 diabetes (2017 Edition) [3]. ACS was diagnosed according to the "Guidelines for diagnosis and treatment of acute ST segment elevation myocardial infarction" (2019) [6], and the " Guidelines for diagnosis and treatment of unstable angina pectoris and acute non ST segment elevation myocardial infarction"((2007) [7], published by Editorial board of Chinese cardiovascular disease Journal and Cardiovascular branch of Chinese Medical Association.

\subsubsection{TCM Discriminate Criteria}

The diagnosis standard for the type of "Qi-Yin deficiency and phlegm-blood stasis" in diabetic chest obstruction, refer to "The new guiding principles of TCM for clinical research" [8].

\subsection{Inclusion Criteria}

The diabetic patients complicated with US, NSTEMI and STEMI, aged between 50 and 75 years, confirmed by coronary angiography as single vessel diameter stenosis $\geq$ $75 \%$, were treated with PCI. Patients whose TCM differentiation conformed to the syndrome of deficiency of Qi-Yin, and internal obstruction by phlegm- blood stasis. patients who were approved by the hospital ethics committee, agreed and signed the informed consent.

\subsection{Exclusion Criteria}

Non diabetic ACS patients for PCI operation. Patients with no Qi-Yin deficiency, no phlegm and blood stasis block; patients with severe heart valve disease, and heart failure (NYHA grade IV); patients with severe liver insufficiency and chronic renal failure.

\subsection{Rejection and Shedding Criteria}

Patients with incomplete data affecting the judgment of curative effect and safety; Patients who quit voluntarily and do not want to continue to cooperate with treatment, and who lose follow-up or with poor compliance; Patients whose condition deteriorate due to other reasons and were in danger of life.

\subsection{Clinical Data}

The 92 cases were all from the inpatients in the department of cardiology and TCM in the Affiliated Hospital of Youjiang Medical College for Nationalities from January 2016 to August 2016, and met the inclusion criteria. There were no significant differences in age, gender, duration of diabetes and $\mathrm{CHD}$, diagnostic constituent ratio, and cardiac function grade between the two groups $(P>0.05)$, and were comparable (Clinical data are shown in Table 1).

Table 1. The comparison of clinical data.

\begin{tabular}{|c|c|c|c|c|c|c|c|}
\hline \multirow{2}{*}{ group } & \multicolumn{3}{|c|}{ cases } & \multirow[b]{2}{*}{ Age (years) } & \multirow{2}{*}{ Diabetic Course (years) } & \multirow{2}{*}{ CHD Course (months) } & \multirow{2}{*}{ BMI $\left(\mathrm{kg} / \mathrm{m}^{2}\right)$} \\
\hline & $\mathbf{n}$ & male & female & & & & \\
\hline control group & 46 & 27 & 19 & $64.44 \pm 11.16$ & $14.31 \pm 5.87$ & $56.22 \pm 15.45$ & $26.85 \pm 1.18$ \\
\hline Treatment group & 46 & 28 & 18 & $65.42 \pm 10.95$ & $13.93 \pm 6.16$ & $55.85 \pm 16.12$ & $26.34 \pm 1.27$ \\
\hline \multirow{2}{*}{ group } & \multicolumn{4}{|c|}{ Diagnosis constitution [cases (\%)] } & & & \multirow{2}{*}{ Cardiac function (NYHA) } \\
\hline & \multicolumn{3}{|c|}{ NSTEAMI } & STEAMI & UAP & Cardiac $\mathrm{f}$ & \\
\hline control group & \multicolumn{3}{|c|}{$17(36.99)$} & $21(45.65)$ & $8(17.39)$ & & $2.13 \pm 0.87$ \\
\hline Treatment group & & $16(34.78$ & & $23(50.00)$ & $7(15.22)$ & \multicolumn{2}{|l|}{$2.22 \pm 0.44$} \\
\hline
\end{tabular}

notes: BMI (Body mass index), NSTEAMI (Non ST elevation myocardial infarction), STEAMI (ST elevation myocardial infarction), UAP (Unstable angina pectoris), NYHA (New York Heart Association). 


\subsection{Therapeutic Method}

The diabetic patients with UAP, STEAMA, NSTEAMI in the two groups were treated according to the treating guidelines for unstable angina pectoris, acute non ST segment elevation myocardial infarction and acute ST segment elevation myocardial infarction [6, 7]. After PCI, Routine treatment is as follows: low molecular heparin calcium injection $(4100 \mathrm{u}$, once a day), oral clopidogrel $(300 \mathrm{mg}$ for the first time, $75 \mathrm{mg}$ after loading dose, once a day), aspirin enteric coated tablets (75mg, once a day), metoprolol (25mg, once a day), benazepril (20mg, once a day), isosorbide mononitrate ( $20 \mathrm{mg}$, twice a day) and Atorvastatin calcium (20mg, once a day); Diabetes is controlled by diet and hypoglycemic therapy according to guideline for prevention and treatment of type 2 diabetes in China [3]. The treatment group were added HJJR, which are composed of ginseng $10 \mathrm{~g}$, astragalus membranaceus $15 \mathrm{~g}$, schisandra chinensis $10 \mathrm{~g}$, ophiopogon japonicus $15 \mathrm{~g}$, cornus officinalis $10 \mathrm{~g}$, yam $15 \mathrm{~g}$, rehmannia glutinosa $15 \mathrm{~g}$, rhubarb $5 \mathrm{~g}$, turtle shell $15 \mathrm{~g}$, cortex moutan $10 \mathrm{~g}$, peach kernel $10 \mathrm{~g}$, salvia miltiorrhiza $10 \mathrm{~g}$, coptis chinensis $8 \mathrm{~g}$, decocted in water for about $300 \mathrm{ml}$, taken three times a day (morning, middle and evening). During the treatment, it is forbidden to use other Chinese medicine for nourishing Yin and tonifying Qi, promoting circulation and removing blood stasis. Appropriate treatment, such as anti-infection treatment for combined infection, could be given to other concomitant diseases, on the premise of not affecting the efficacy of the trial drug. The treating course were two months.

\subsection{Cardiac Function Efficacy Judgment}

Cardiac function was recorded according to NYHA grade I-IV, and the curative effect of cardiac function was evaluated according to the guiding principles for clinical research of new TCM [8]. Remarkable effect: heart failure is effectively controlled and cardiac function is improved by more than grade 2; Effective: the symptoms of heart failure were improved, and the cardiac function was improved by grade 1, but less than grade 2; Ineffective: the symptoms of heart failure did not improve, or the improvement of cardiac function was less than grade 1; Deterioration: the deterioration of cardiac function is grade 1 or above.

\subsection{Detection of Serum Inflammatory Factors and Ang II}

On the morning of admission and discharge, $6 \mathrm{ml}$ fasting venous blood of the two groups were taken and placed in the anticoagulant tube containing EDTA. After centrifugation (2500 $\mathrm{r} / \mathrm{min})$, the supernatant were collected and placed in $-80^{\circ} \mathrm{C}$ refrigerator for frozen storage. The C-reactive protein (CRP), IL-6, TNF- $\alpha$ and Angiotensin II (Ang II), were determined with enzyme-linked immunosorbent assay (ELISA), whose kit were provided by Wuhan doctor de Bioengineering Co., Ltd, by which instructions the specific testing steps were conducted out.

\subsection{Detection of Cardiac Remodeling Index}

Before and after 2 months treatment, the patients were examined by echocardiography (using German Siemens C2000 color Doppler diagnostic instrument, phased array probe, probe frequency of $3.5 \mathrm{MHz}$ ), and the morphological indexes of left ventricle [left ventricular end diastolic diameter (LVEDD), left ventricular end systolic diameter (LVESD), Left ventricular end-diastolic ventricular septal thickness (LVEST), Left ventricular posterior wall thickness (LVPWT)], and the Left ventricular function index [Left ventricular fractional shortening $(\Delta \mathrm{FS})$, left ventricle ejection fraction (LVEF), stroke volume (SV), cardiac output (CO), cardiac index (CI), maximum blood flow velocity of early diastolic/atrium late diastolic (E/A)] were detected.

\subsection{Statistical Methods}

All the data were analyzed by SPSS17.0 medical statistical software. The measurement data were expressed as $(\bar{x} \pm \mathrm{S})$, and the paired t-test were used to compare the data before and after clinical trials, while the t-test in two independent samples was used for the comparison between groups. The count data were expressed as $\%$ and tested by $\mathrm{x}^{2} . P<0.05$ was significant statistically.

\section{Results}

\subsection{Comparison of Cardiac Function Between the Two Groups After Treatment}

Post-therapy, the total effective rate and 5-year survival ratio in treatment group were significantly higher than those in control group $(\mathrm{P}<0.05)$.

Table 2. Comparison of cardiac function and prognosis between the two groups after treatment [cases (\%)].

\begin{tabular}{llllllll}
\hline group & $\mathbf{n}$ & Remarkable effect & Effective & invalid & deteriorate & Total effective & 5-year survival \\
\hline control group & 46 & $11(23.91)$ & $20(43.48)$ & $9(19.57)$ & $6(13.04)$ & $31(67.39)$ & $38(82.61)$ \\
Treatment group & 46 & $15(32.61)$ & $25(54.35)$ & $3(6.52$ & $2(4.34)$ & $40(86.96)^{\Delta}$ & $44(95.53)^{\Delta}$ \\
\hline
\end{tabular}

Note: compared with control group, ${ }^{\Delta} P<0.05$.

\subsection{Comparison of Serum Mediators CRP, IL-6, TNF- $\alpha$, Ang II Before and After Treatment}

Compared with pre-treatment, the serum inflammatory mediators CRP, IL-6, TNF- $\alpha$ and Ang II all decreased in the treatment group and the control group. Comparison between groups, the serum inflammatory mediators and Ang II decreased more obvious, with significant discrepancy $(\mathrm{P}<0.05)$. The results are shown in Table 3 . 
Table 3. Comparison of serum mediators CRP, IL-6, TNF- $\alpha$, Ang II before and after treatment in the two groups ( $\bar{x} \pm s)$.

\begin{tabular}{|c|c|c|c|c|c|c|}
\hline Groups & & $\mathbf{n}$ & Ang II (ng/L) & IL-6 (ng/L) & TNF- $\alpha(n g / L)$ & CRP (mg/L) \\
\hline \multirow{2}{*}{ Treatment group } & Before treatment & 46 & $101.84 \pm 28.50$ & $174.37 \pm 36.86$ & $46.12 \pm 10.41$ & $9.32 \pm 2.67$ \\
\hline & After treatment & 46 & $72.98 \pm 18.53^{* \Delta}$ & $128.72 \pm 32.79^{* \Delta}$ & $28.45 \pm 7.55^{* \Delta}$ & $4.69 \pm 1.02^{* \Delta}$ \\
\hline \multirow{2}{*}{ control group } & Before treatment & 46 & $102.58 \pm 27.65$ & $172.47 \pm 37.05$ & $45.54 \pm 11.25$ & $9.47 \pm 2.21$ \\
\hline & After treatment & 46 & $83.47 \pm 22.39^{*}$ & $144.48 \pm 37.69^{*}$ & $36.14 \pm 13.83^{*}$ & $7.98 \pm 2.16^{*}$ \\
\hline
\end{tabular}

Note: compared with before treatment, ${ }^{*} P<0.05$; compared with the control group, ${ }^{\Delta} P<0.05$

\subsection{Comparison of Cardiac Remodeling Indexes Between the Two Groups Before and After Treatment (Table 4)}

Compared with before treatment, the difference of $\triangle \mathrm{FS}, \mathrm{SV}$, $\mathrm{CO}, \mathrm{CI}, \mathrm{LVEF}$ and $\mathrm{E} / \mathrm{A}$ in the treatment group were statistically significant $(\mathrm{P}<0.05, \mathrm{P}<0.01)$. LVEDD, LVESD, LVEST, LVPWT increased slightly, but the difference were not statistically significant; while there were significant differences in LVEDd, LVESD, SV, CI, LVEF and $\mathrm{E} / \mathrm{F}$ in control group $(\mathrm{P}<0.05)$.

After treatment, the LVEDd and LVESD in treatment group were lower than those in control group $(\mathrm{P}<0.05)$; while the $\Delta$ FS, SV, CO, CI, LVEF and E / A were significantly higher than those in the control group $(\mathrm{P}<0.05, \mathrm{P}<0.01)$.

Table 4. Comparison of cardiac remodeling indexes in the two groups before and after treatment.

\begin{tabular}{llllllll}
\hline Groups & & $\mathbf{n}$ & LVEDD $(\mathbf{m m})$ & LVESD $(\mathbf{m m})$ & LVEST $(\mathbf{m m})$ & LVPWT $(\mathbf{m m})$ & $\Delta$ FS $(\%)$ \\
\hline Treatment & Before treatment & 46 & $46.58 \pm 2.97$ & $32.85 \pm 2.87$ & $8.86 \pm 1.31$ & $9.85 \pm 1.38$ & $23.85 \pm 3.18$ \\
group & After treatment & 46 & $51.85 \pm 2.82^{\Delta}$ & $36.85 \pm 3.68^{\Delta}$ & $11.22 \pm 1.53$ & $10.82 \pm 1.25$ & $32.86 \pm 5.02^{\star \Delta}$ \\
control & Before treatment & 46 & $46.75 \pm 3.16$ & $33.04 \pm 2.76$ & $9.02 \pm 1.35$ & $9.79 \pm 1.41$ & $23.08 \pm 3.44$ \\
group & After treatment & 46 & $58.45 \pm 2.93^{\text {市}}$ & $41.85 \pm 3.45^{\text {市}}$ & $11.68 \pm 1.43$ & $11.13 \pm 1.38$ & $26.73 \pm 4.86$ \\
\hline
\end{tabular}

\begin{tabular}{|c|c|c|c|c|}
\hline SV (ml) & $\mathrm{CO}(\mathrm{L} / \mathrm{min})$ & $\mathrm{CI}\left(\mathrm{ml} / \mathrm{s} \cdot \mathrm{m}^{2}\right)$ & LVEF (\%) & $\mathbf{E} / \mathbf{A}$ \\
\hline $40.78 \pm 5.63$ & $3.22 \pm 0.48$ & $40.91 \pm 6.68$ & $45.85 \pm 3.66$ & $0.65 \pm 0.14$ \\
\hline $68.77 \pm 4.28^{\star \Delta}$ & $5.87 \pm 0.82^{\star \Delta}$ & $55.56 \pm 8.82^{\star \Delta}$ & $59.77 \pm 3.94^{\star \Delta}$ & $1.48 \pm 0.17^{\text {ॠ } \Delta}$ \\
\hline $41.07 \pm 5.37$ & $3.34 \pm 0.41$ & $41.35 \pm 6.43$ & $46.03 \pm 3.68$ & $0.68 \pm 0.16$ \\
\hline $48.68 \pm 3.86^{\text {म }}$ & $5.21 \pm 0.38$ & $47.85 \pm 8.38^{\text {市 }}$ & $51.96 \pm 3.82^{\text {r }}$ & $1.08 \pm 0.19^{\text {㑞 }}$ \\
\hline
\end{tabular}

Note: compared with before treatment, ${ }^{\star} P<0.05,{ }^{\star} P<0.01$; compared with the control group, ${ }^{\wedge} P<0.05,{ }^{\wedge} P<0.01$

\subsection{Adverse Reactions}

During the treatment, there were no significant abnormal changes in liver and renal function, and there were no abnormalities and adverse reactions in routine examination of blood, urine and stool, which showed that Huoxue Jiedu Jiangtang Recipe were safe.

\section{Discussion}

In recent years, it has been found that there is no direct relationship between coronary stenosis and myocardial ischemia, and that revascularization (such as PCI) can only relieve coronary stenosis but not cure myocardial ischemia [9]. There are many causes for ischemic heart disease, and coronary stenosis is only one of them, other causes include spontaneous thrombosis, spasm, inflammation, microvascular dysfunction, endothelial dysfunction and neovascularization $[10,11]$. Myocardial ischemia activates sympathetic nervous system and renin-angiotensin-aldosterone system (RAAS), In which activation of sympathetic nerve can result in excessive release of catecholamine in cardiac tissue, which directly damages cardiac myocytes and cardiac function, necrosis and apoptosis of cardiac myocytes, leading to myocardial fibrosis, and promoting ventricular remodeling; while RAAS activation can increase Ang II in local myocardium, aldosterone synthesis, collagen producing, myocardial fibrosis and myocardial tissue remodeling [6, 7]. Myocardial ischemia and necrosis can produce necrotic substances and inflammatory reaction, which can make myocardial fibrosis, cardiomyocyte hypertrophy and scar formation, contributing to cardiac remodeling. Ischemic (or necrotic) myocardium can also lead to uncoordinated ventricular diastolic and systolic activities, hold back ventricular ejection function, decrease cardiac output and increase left ventricular end diastolic pressure, resulting in ventricular dilation and distortion and aggravating cardiac remodeling [12].

Coronary heart disease in the general population often presents a coronary stenosis, which can be reconstructed by PCI, and on the basis of standardized treatment of Western medicine, the quality of life can often be improved, the hospitalization and mortality rate are often reduced, the prognosis are greatly improved [13]. But with regard to the diabetes population, the duration onset of diabetes and the level of blood sugar are the risk factors of prognosis in diabetic CHD [14]. Diabetic patients often suffer from coronary endothelial dysfunction and decreased coronary reserve, which may lead to abnormal myocardial perfusion. Therefore, ischemic heart disease is more common and serious in diabetes than in non-diabetic patients [15]. What is more, diabetic coronary artery disease is more common with multiple, diffuse and small vessel diseases. Multivessel stenosis can lead to widespread myocardial ischemia, while the extensive stenosis of small arteries is extremely 
unfavorable to the establishment of collateral circulation and reperfusion [16]. Therefore, in addition to the proximal coronary artery injury, diabetic patients tend to have diffuse distal injuries and microvascular occlusions, so that the collateral circulation cannot be established in time, which aggravate myocardial necrosis, cardiac remodeling and cardiac function deterioration. Compared with general patients, the prognosis of patients with diabetic myocardial infarction is not due to massive myocardial infarction. MILIS data show that although most patients are small focal myocardial infarction, the prognosis of patients with diabetes are worse than common type of patients (4 years mortality: $25.9 \%$ VS 14.5\%) [17]. After PCI, although the infarcted vessels are recanalized, there are still many residual stenosis, which is adverse to myocardial reperfusion. Therefore, for diabetic patients with acute coronary syndrome, there are still so a large residual risk after PCI that limit the treatment of Western medicine alone. Combination of traditional Chinese and Western medicine can rationally prescribe medication according to the characteristics of diabetes, which is different from that of the general population.

According to TCM theory, Yin debilitation and Qi deficiency, Combined with dryness-heat, are the Diabetes mellitus (Xiao $\mathrm{Ke}$ ), in which, with the development of the disease, heart Qi unable to promote the blood circulation, leading to Qi stagnation and blood stasis; Or the accumulation of internal heat damage body fluid, transform into fire and result in internal exuberance of fire-heat that may in turn consume or scorch the body Yin-fluids and blood, and subsequently lead to blood and fluid stasis and change into stasis toxin [18]. Therefore, "Qi-Yin deficiency, blood stasis and toxin accumulation" can develop into the diabetic CHD pathogenesis [18]. Although the stent placement can reopen the coronary arteries for diabetic patients with acute coronary syndrome (ACS), it cannot remove the lesions, and the atherosclerotic plaques still exist. What is more, stent implantation can cause a new damage to the coronary artery, which will aggravate the inflammation and trigge the blood stasis [19]. The essences of the deficiency of Qi and Yin and the stagnation of phlegm and stasis toxin, are still the etiology and pathogenesis of ACS after PCI. Scientific researches have certified that, atherosclerosis is a process of artery post-injury inflammation, in which a large number of inflammatory cytokines accumulate with active metabolism, where local plaque temperature were generally higher than the surrounding artery, and were positively correlated with plaque instability, consequently, after PCI, inflammation and heat release increase in myocardial infarction [20,21]. The essential characteristics of inflammatory reaction are similar to heat-toxin struggles, suggesting that heat clearing and detoxifying drugs can protect the heart. Therefore, the management for diabetic ACS patients, their perioperative or postoperative long-term therapy should focus on the basic pathogenesis of heat toxin, and attach importance to clearing away heat and detoxification.

HJJR compose of turtle shell, ginseng, astragalus, ophiopogon japonicus, radix rehmanniae, fructus corni, rhubarb, salvia miltiorrhiza, rhizoma coptidis, cortex moutan, peach kernel, yam and fructus schisandrae chinensis, in which, cornus, rehmannia and yam play the roles of nourishing kidney, and filling Yin, for treating true Yin deficiency; ginseng, schisandra, ophiopogon root, possess efficency of tonifying Qi, nourishing Yin, generating fluid, and nourishing heart; turtle shell, peach kernel, rhubarb and cortex moutan are Eastern Han Dynasty medical expert Zhang Zhongjing's "Turtle shell pills" ingredients, can nourish kidney Yin, clear away asthenia heat, sharing dissipating stagnation and detoxification, for treating "Accumulation"; salvia miltiorrhiza coordinated with cortex moutan and peach kernel, can promote blood circulation; rhubarb combined with hizoma coptidis can eliminate heat-toxin and dispell dampness; ginseng cooperated with astragalus membranaceus, can invigorate Qi and generate blood, making toxins floated and dissipated. All prescriptions share supplementing and attacking in combination [4].

\section{Conclusion}

This study found that, in diabetic patients with acute coronary syndrome after PCI, basis on conventional western medicine treatment plus HJJR, the left ventricular morphology index LVEDD and LVESD were significantly reduced, while the cardiac function index LVEF, E / A, SV, $\mathrm{CO}, \mathrm{CI}$ and $\Delta \mathrm{FS}$ were significantly increased; the levels of inflammatory mediators IL-6, CRP, TNF- $\alpha$ and serum Ang II were decreased. The results indicate that, by alleviating the inflammatory response caused by oxidative stress of diabetes mellitus, inhibiting the RASS system, HJJR can improve ventricular remodeling, enhance heart function, improve the quality of life and prognosis, with good patient compliance and less adverse reactions. This study suggests that the prevention and treatment of heart failure should not only rely on the improvement of hemodynamics, but also pay attention to combating myocardial remodeling, inhibiting the over activation of pro-inflammatory factors and antagonizing the over secretion of neuro-endocrine system. Compared with ordinary patients, diabetic patients with CHD can still deteriorate the illness after PCI, facing the progress of cardiac remodeling, eventually leading to heart failure, duo to coexisting diabetes mellitus. Therefore, multi-target and multi-channel treatments are the main idea of prevention and treatment in the future, which is the advantage of TCM.

At present, at home and abroad, although the guidelines for CHD clinical diagnosis and treatment have been established, there are still individual differences in clinical practice. For diabetic groups, a variety of pathological factors can aggravate $\mathrm{CHD}$, result in progression, poor prognosis and low survival rate. In the future, with the continuous development of medical technology, the indications of PCI technology will constantly broaden from unsuitable to suitable, so that the PCI patients will continue to increase, with the residual risk of Western medicine treatment increasing, the challenges in postoperative treatment growing. Although PCI has played a certain role in CHD, the intrinsic nature of the deficiency Qi-Yin, and the accumulation of 
blood stasis-toxin, are still the pathogenesis of postoperative PCI. Consequently, "Double regulation of TonifyingDetoxifing" exert TCM unique advantage of whole regulation and intervention of multi-way, multi-link and multi-target, supplementing to modern routine medicine. Therefore, in the future, we need to conduct a multicenter, large sample, randomized controlled clinical trial, and introduce long-term follow-up studies. At the same time, we will further study the HJJR pharmacological mechanism, so that the characteristics of TCM differential treatment, dynamic adjustment, and overall intervention can be fully demonstrated.

\section{Acknowledgements}

This research is supported by National Natural Science Foundation of China (81960833); Guangxi emphasis research and development program (2017AB45042); Guangxi Natural Science Foundation (2019JJA140146).

\section{References}

[1] Kai Yu, Liyun Ma, Yingping Wei. The Risk of Cardiovascular Events in Patients with Coronary Heart Disease Combined with Type 2 Diabetes: Meta-Analysis. Advances in Clinical Medicine. 2021, 11 (1): 125-131.

[2] Wan Chunling, Yang Yaru, Jiao Yahui. Clinical analysis of adverse cardiovascular events in patients with acute coronary syndrome complicated with type 2 diabetes mellitus. J Clin Psychosom Dis, 2018, 24 (4): 20-22.

[3] Diabetes branch of Chinese Medical Association. Guidelines for the prevention and treatment of type 2 diabetes in China. Chinese Journal of diabetes. 2018, 10 (1): 4-65.

[4] Fu Xianzhao, Huang Zhenfeng, Huang Wenhua, et al. Review and Prospect of the Preventive Effect of "Supplementing Qi and Nourishing Yin, Activating Blood Circulation and Detoxifying" on Diabetes Mellitus Complicated with Acute Coronary Syndrome. [J]. Chin J TCM WM Crit Care, 2017, 24 (5): 547-551.

[5] Fu Xianzhao*, Huang GuangMing, Qiu HaiXian, et al. Immune Regulation of "Nourishing Yin and Qi, Activating Blood Circulation and Detoxifying" in Elderly Diabetic Patients omplicated with Coronary Heart Disease. Science Journal of Public Health, 2020, 8 (6): 161-167.

[6] Cardiovascular branch of Chinese Medical Association, Editorial board of Chinese Journal of cardiovascular disease. 2019 Chinese Society of Cardiology (CSC) guidelines for the diagnosis and management of patients with ST-segment elevation myocardial infarction. Chin J Cardiol, 2019, 47 (10): 766-783.

[7] Cardiovascular branch of Chinese Medical Association, Editorial board of Chinese Journal of cardiovascular disease. Unstable angina and non-ST-elevation myocardial infarction guidelines for diagnosis and treatment. Chin J Cardiol, 2007, 35 (4): 295-304.
[8] Zheng Xiaoyi. the new guiding principles of Chinese medicine for clinical research. BeiJing: China Medical Science and Technology Publishing House, 2002: 68-73.

[9] ZHONG Qiaoqing, ZHU Lingyan, ZHANG Guogang, et al. Atherosclerotic cardiovascular disease in diabetes--mechanisms, clinical represent and management. Chinese Journal of Cardiovascular Review, 2018, 16 (12): 1069-1072.

[10] Shen Ying, Ding Fenghua, Lu Lin, Zhang Ruiyan, Shen Weifeng. Blood pressure management in diabetic patients with coronary artery disease. Chinese Journal of Cardiology, 2020, 1: $5-9$.

[11] CZHANG Zengping, WANG Weijia, KONG Yina, WANG Congrui. orrelation between the stability of carotid artherosclerotic plaque and the levels of serum adopinectin and IL-8 in patients with acute cerebral infraction. J Xuzhou Med Univ 2, 2019, 39 (4): 289-292.

[12] Zeinalova NV, Kurbanov YZ, Mirzazade VA, Rzayeva RA, Novruzova MS. The risk of cardiovascular death in type 2 diabetes. Klin Med (Mosk), 2017, 95: 57-59.

[13] Gao Runlin. Advances and perspectives in coronary intervention in China. Chin J Cardiol, 2019, 47 (9): 675-679.

[14] Buntaine AJ, Shah B, Lorin JD, Sedlis SP. Revascularization Strategies in Patients with Diabetes Mellitus and Acute Coronary Syndrome [J]. Curr Cardiol Rep, 2016, 18 (8): 79.

[15] Hong tianpei, Mu yiming, Zhu, dalong. Intervention for adults with pre-diabetes: A Chinese expert consensus. Chinese Journal of Endocrinology and Metabolism, 2020, 5: 371-380.

[16] LU Hong-yan, WAN Min-ying, WANG Cheng. Analysis of Clinical Characteristics of Patients with Diabetes Mellitus Complicated with Coronary Heart Disease and Acute Myocardial Infarction. China \&Foreign Medical Treatment, 2021, 7: 88-90.

[17] Michaelael TJ, Aristidis V. Diabetes and cardiovascular disease [M]. Sun FL translation. Ji Nan: Shandong science and technology press, 2008, 430-431.

[18] FU Xian-zhao, XU Jing, HUANG Wen-hua, et a1. Strategy of Stasis-resolving and Detoxification on Diabetic Coronary Heart Disease [J]. JETCM, 2014, 23 (11): 2024-2027.

[19] ZHU Yun-qing, HU Yun, MAO Xiao-ming. The research progress of immune disorders in Type 2 diabetes mellitus. Chinese Journal of Diabetes, 2015, 23 (9): 859-861.

[20] DANG Jingyi, HAO Qimeng, JIANG Na, et al. Relationship between serum lipoprotein (a), $\beta 2$-microglobulin and tumor necrosis factor- $\alpha$ levels and degree of coronary artery lesion in patients with acute coronary syndrome. Chin J Arterioscler, 2020, 28 (8): 702-706.

[21] Wang Ying, Wang Aihua, Tian Tian. Correlation of endothelin-1, adiponectin, C-reactive protein and related metabolic indexes in type 2 diabetes mellitus with atherosclerosis. Guangdong Medical Journal, 2019, 40 (7): 1020-1024. 